\title{
Short Term Outcomes of Head and Neck Oncology Surgery During Covid-19 Pandemic: Experience from a Tertiary Cancer Care Centre in North India
}

\author{
Sumeet Jain $^{1}\left(\mathbb{D} \cdot\right.$ Sumit Gupta $^{1} \cdot$ Tejinder Pal Singh ${ }^{2} \cdot$ Kavita Chhabra $^{3}$. \\ Richa Jain $^{3}$ - Akashdeep Singh Sohi ${ }^{1}$ - Deepender Kaur Chhina ${ }^{4}$ Gurpreet Singh Brar ${ }^{1}$. \\ Rajinder Kumar Mittal ${ }^{5} \cdot$ Parshotam Lal Gautam $^{2}$
}

Received: 4 November 2020/Accepted: 14 December 2020/Published online: 8 January 2021

(C) Association of Otolaryngologists of India 2021

\begin{abstract}
With the entire world in the midst of COVID-19 pandemic, several health care facilities have stopped or delayed performing elective surgeries in order to cater to ever increasing number of COVID-19 patients. Moreover, there were initial reports of poor surgical outcomes in patients who underwent surgery and were found to be positive for COVID-19 infection in post-operative period. In this study, we have evaluated the short-term outcomes of head and neck oncology patients operated in our institute following a strict screening protocol and conducting COVID-19 testing by Reverse transcriptase polymerase chain reaction once the test was available. 68 patients operated between 1st April and 30th September, 2020 (COVID-19 era, study group) were compared with 59 patients operated during 1st October, 2019 to 31st March 2020 (Non COVID-19 era, control group). The comparison between the groups was done by measuring 30 days complication rate as defined by Dindo-Clavien classification. $10.3 \%$ of patients developed complications in study group as compared to $8.5 \%$ of patients in control group
\end{abstract}

Sumeet Jain

sumeetpublish@gmail.com

1 Department of Surgical Oncology, DMCH Cancer Care Centre, Civil Lines, DMC Road, Tagore Nagar, Ludhiana, Punjab 141001, India

2 Department of Critical Care Medicine, Dayanand Medical College and Hospital (DMCH), Ludhiana, India

3 Department of Anaesthesia, Dayanand Medical College and Hospital (DMCH), Ludhiana, India

4 Department of Microbiology, Dayanand Medical College and Hospital (DMCH), Ludhiana, India

5 Department of Plastic Surgery, Dayanand Medical College and Hospital (DMCH), Ludhiana, India which was statistically non-significant $(p=0.7)$. Importantly, none of the patients developed any sign or symptom suggestive of COVID-19 infection in post-operative period in study group. Head and neck oncology related cancer procedures including complex reconstruction can safely be performed during COVID-19 era by proper screening and pre-operative testing for COVID-19. We also suggest use of N95 masks and face shields as bare minimum in order to ensure the safety of health care workers even after a negative COVID-19 report.

Keywords Head and neck cancer surgery · COVID-19

\section{Introduction}

The entire world is in the midst of coronavirus 19 (COVID19) pandemic. It has put immense pressure on healthcare facilities with several centres have stopped or delayed doing elective surgeries [1]. But any delay in oncological procedures can lead to disease progression and affect patient outcome. Recent international guidelines recommend to operate most of the head and neck oncology patients within 8 weeks from diagnosis [2]. Management of head and neck cancer patients poses a greater risk of exposure of COVID-19 to healthcare workers than any other cancer site. This is because, firstly it involves working near the sites of viral replication viz nasal cavity, oropharynx and nasopharynx, beginning from the pre-operative diagnostic procedures to intra-operative and postoperative period. Secondly, head and neck oncologic surgeries involve procedures which are considered high risk for viral aerosolization and can cause transmission of infection to those present in operating room [3]. This risk continues even in the post-operative period especially in 
the patients who are tracheostomized or have undergone laryngectomies [3]. Moreover, there are reports of poor surgical outcomes and increased post-operative mortality in patients who had COVID-19 infection [4].

In our study, we wish to share our experience regarding the short term surgical outcomes of head and neck cancer patients and the protocols being followed by us to ensure the safety of our patients as well as health care workers involved in the care of these patients.

\section{Materials and Methods}

Patients of head and neck cancer operated under general anaesthesia from 1st October, 2019 to 30th September 2020 were included in the study. Patients operated from 1st April, 2020 to 30th September, 2020 constituted the study group (cases operated during COVID-19 pandemic) and patients operated between 1st October, 2019 and 30th March, 2020 constituted the control group (cases operated in non-COVID-19 era). The data was obtained retrospectively from our prospectively maintained database. Preoperative COVID-19 testing was done in all patients starting from mid of May, 2020. Sixty-nine patients were evaluated for surgery during COVID-19 time and there were 59 patients in pre COVID-19 era. Out of 69 patients in COVID-19 era 66 patients underwent pre-operative testing by quantitative RT-PCR (Reverse transcriptase polymerase chain reaction) either as out-patient or in a designated pre-COVID ward. Only the patients with negative COVID-19 report were admitted in our regular ward. The report was not more than 5 days old on the day of surgery, the date calculated from time of sample collection and not the reporting date. The patients were also advised to self-isolate at home in case COVID-19 test was done on out-patient basis. We continued to follow the treatment protocol as was being given in pre-COVID era. The use of N95 mask and face shield was mandatory for all the health care workers involved in care of these patients. The objective of the study was to find out whether the shortterm outcome of patients which were properly screened and tested for COVID-19 infection was different from those operated during non-pandemic era. This was done by measuring 30 days complication rate as defined by DindoClavien classification in the two groups [5].

\section{Statistical Analysis}

The Statistical Product and Service Solutions, SPSS 21 for windows was used for performing statistical analysis. Pearson Chi square test was used for statistical analysis of categorial data and $t$ test was used for comparison of means of two groups. As for majority of statistical analysis, $p$ value $<0.05$ was taken as statistically significant.

\section{Results}

Sixty-nine patients were evaluated for surgery during COVID-19 pandemic. All these patients were asymptomatic for any sign or symptom of COVID-19 infection. Out of these 66 patients underwent pre-operative testing for COVID-19 and one patient tested positive for the disease. The surgery was deferred for the patient testing positive. So, finally there were 68 patients who underwent surgery during COVID-19 era (study group) and 59 patients in nonCOVID-19 era (control group).

The study and control groups were similar with respect to mean age and presence of co-morbidities (Table 1). Oral cavity cancer was the most common site operated in both the groups (60\% in study group vs. $66 \%$ in control group). The groups were also similar with respect to duration of surgery with majority of surgeries in both groups lasting more than $4 \mathrm{~h}$ (Table 1). Reconstruction either in the form of pedicle or free flaps was required in about $44 \%$ of patients in study group and 39\% of patients in control group. With respect to the type of re-construction used, both groups had similar number of free flaps but more patients in study group (21 patients) underwent pedicle flaps as compared to control group (13 patients) (Table 1). $10.3 \%$ of patients developed complications in study group as compared to $8.5 \%$ of patients in control group which was statistically non-significant $(p=0.7)$ (Table 1). Grade III and IV complications occurred in 6 patients in study group and in 4 patients in control group (Table 2). None of the patients in study group developed any signs or symptoms suggestive of COVID-19 infection during post-operative period. Four of our healthcare workers involved in the care of our patients tested positive for COVID-19 infection but source of contact for all these workers were traced to be external rather than any of the patients.

\section{Discussion}

Our set up is a dedicated cancer institute housed in a separate building within the campus of more than 1500 bedded tertiary care hospital and medical college. We have our own separate operation theatres, intensive care beds and in-patient beds. This has enabled us to continue our cancer related services even during COVID-19 period. There was initial slowdown in number of patients presenting to us but it has gradually picked up and has maintained a steady flow after that (Table 3). There were initial recommendations of avoiding complex 
Table 1 Demographic and clinical details of the two groups

\begin{tabular}{|c|c|c|c|}
\hline & Study group $(n=68)$ & Control group $(\mathrm{n}=59)$ & $p$ value \\
\hline Age (years) & 52.7 & 52.2 & $p=0.8$ \\
\hline Male & $45(66.2 \%)$ & $38(64.4 \%)$ & $p=0.8$ \\
\hline Female & $23(33.8 \%)$ & $21(35.6 \%)$ & \\
\hline \multicolumn{4}{|l|}{ Co-morbidity } \\
\hline Present & $16(23.5 \%)$ & $18(30.5 \%)$ & \multirow[t]{2}{*}{$p=0.3$} \\
\hline Absent & $52(76.5 \%)$ & $41(69.5 \%)$ & \\
\hline \multicolumn{4}{|l|}{ Site } \\
\hline Oral cavity & $41(60.3 \%)$ & $39(66.1 \%)$ & \multirow[t]{5}{*}{$p=0.1$} \\
\hline Laryngopharynx & $7(10.3 \%)$ & $5(8.5 \%)$ & \\
\hline Thyroid & $8(11.8 \%)$ & $12(20.3 \%)$ & \\
\hline Parotid & $5(7.4 \%)$ & $1(1.7 \%)$ & \\
\hline Miscellaneous & $7(10.3 \%)$ & $2(3.4 \%)$ & \\
\hline \multicolumn{4}{|l|}{ Duration of surgery } \\
\hline$<2 \mathrm{~h}$ & $12(17.6 \%)$ & $8(13.6 \%)$ & \multirow[t]{3}{*}{$p=0.7$} \\
\hline $2-4 \mathrm{~h}$ & $25(36.8 \%)$ & $25(42.4 \%)$ & \\
\hline$>4 \mathrm{~h}$ & $31(45.6 \%)$ & $26(44.1 \%)$ & \\
\hline \multicolumn{4}{|l|}{ Reconstruction } \\
\hline Primary closure & $38(55.9 \%)$ & $36(61 \%)$ & \multirow[t]{3}{*}{$p=0.5$} \\
\hline Pedicle flaps & $21(30.9 \%)$ & $13(22 \%)$ & \\
\hline Free flaps & $9(13.2 \%)$ & $10(16.9 \%)$ & \\
\hline \multicolumn{4}{|l|}{ Tracheostomy } \\
\hline Yes & $17(25 \%)$ & $12(20.3 \%)$ & \multirow[t]{2}{*}{$p=0.5$} \\
\hline No & $51(75 \%)$ & $47(79.7 \%)$ & \\
\hline \multicolumn{4}{|c|}{ Post-operative complications } \\
\hline Yes & $7(10.3 \%)$ & $5(8.5 \%)$ & \multirow[t]{2}{*}{$p=0.7$} \\
\hline No & $61(89.7 \%)$ & $54(91.5 \%)$ & \\
\hline
\end{tabular}

Table 2 Classification of complications (according to Dindo-Clavien classification [5])

\begin{tabular}{lll}
\hline & Study group $(\mathrm{n}=68)$ & Control group $(\mathrm{n}=59)$ \\
\hline Complication & $7(10.3 \%)$ & $5(8.5 \%)$ \\
Grade I & Oro-cutaneous fistula-1 & Oro-cutaneous fistula-1 \\
Grade II & None & None \\
Grade IIIa & Oro-cutaneous fistula requiring re-exploration under local anaesthesia—-1 & None \\
Grade IIIb & Re-exploration free flap related vascular problem-2 & Re-exploration free flap related vascular problem- \\
& & 2 \\
& Re-exploration for bleeding-1 & Re-exploration for bleeding-1 \\
& Oro-cutaneous fistula requiring re-exploration under general anaesthesia— & \\
Grade IVa & Pulmonary thromboembolism-1 & \\
\hline
\end{tabular}

microvascular reconstructions and instead perform local or regional flaps so as to reduce operation theatre time and to avoid surgeries requiring elective tracheostomies which generate considerable aerosols [1]. However, considering the fact that head and neck oncological surgeries cannot be postponed indefinitely, recent international recommendations still favours regional or local flaps over free flaps but are not in favour of avoiding tracheostomies if deemed 
Table 3 Month wise distribution of cases operated during COVID19 era

\begin{tabular}{lc}
\hline Month (2020) & Number of patients operated \\
\hline April & 2 \\
May & 13 \\
June & 21 \\
July & 10 \\
August & 11 \\
September & 11 \\
\hline
\end{tabular}

necessary [2]. With regards to free flap reconstruction, we too have been inclined to do more pedicle or local flaps but have done free flaps as and when required (Table 1).

There were initial reports of poor surgical outcomes in oncology patients who tested positive for COVID-19 in post-operative period [4]. We have been following strict symptom-based screening in initial period of pandemic when the test was not available. With availability of testing, all our pre-operative patients were tested before surgical procedure. In our study, the short-term outcome of our patients operated in COVID-19 era were similar to those operated in non-COVID-19 era as measured by 30-day complication rate (Table 1). None of our patients required post-operative ventilation. Importantly, there were no post-operative pulmonary complications related to COVID-19 infection or mortality in patients operated during COVID-19 era. One patient operated in study group had radiologically documented pulmonary thromboembolism (Table 2). This patient required ICU care and inotropic supports but gradually recovered and was discharged. Similarly, low rate of post-operative complications in elective head and neck cancer patients was reported in another study from Europe by Brar et al. [6]. In this study, the authors concluded that with pre-operative screening of patients, head and neck cancer surgery can be performed safely during the pandemic [6]. A study conducted by Nekkanti et al. at Tata Memorial Hospital; Mumbai also suggested that pre-operative testing has a beneficial role in giving good post-operative outcomes [4]. There was also a strong recommendation for doing the same by a recent consensus guideline for head and neck cancer management [2]. In our study, 95.7\% (66 out of 69 patients) underwent pre-operative testing with only one patient $(1.5 \%)$ tested positive for the disease. Importantly, none of the patients in our study developed any signs or symptoms suggestive of COVID-19 requiring testing during post-operative period. This was also the case in study reported by Nekkanti et al. where none of the patients developed any signs of COVID-19 infection [4].
Though a negative report of COVID-19 test by RT-PCR is reassuring for both patients and health care workers, however, it has a limitation that this test has been reported to have false negative rate ranging from 16 to $24 \%$ in symptomatic patients which may be higher in case of asymptomatic patients [3]. We haven't had any patient so far who have tested positive in post-operative period but can happen in near future. Keeping this in perspective, so even with a negative COVID-19 report, all our health care workers involved in care of patients were advised to use N95 and protective eye covering (in form of googles or face shields). There are no consensus guidelines regarding what constitutes an ideal protection equipment while operating patients who have negative COVID-19 report with most of the institutes having their own institutional policies. Nekkanti et al., in their study of pre-operative COVID-19 testing too have stressed on the need of highquality masks and other protocols viz social distancing, hand hygiene etc. for prevention of COVID-19 infection [4]. We too have followed the above policy and it seems to be working well for us till now. The use of these protective gears may lead to impairment in communication and visibility (mainly due to fogging of googles) but one has to get used to this new normal till the time we have an effective vaccine or the pandemic ends [7].

\section{Conclusion}

Head and neck oncology related cancer procedures including complex reconstruction can be safely performed during COVID-19 era without increase in post-operative complications by proper screening and pre-operative testing for COVID-19 ( RT-PCR) resulting surgical outcomes comparable to pre-COVID-19 era. We suggest use of N95 masks and face shields as bare minimum in order to ensure the safety of health care workers even after a negative COVID-19 report. Further studies forthcoming in this regard may enlighten us to what may be the optimal guidelines for pre-operative testing of patients and adequate personal protection equipment for health care workers.

Acknowledgements We acknowledge the contribution Department of Microbiology, Dayanand Medical College for their contribution in successfully establishing the facility for COVID-19 infection and tireless processing samples of our patient's day and night.

Author's Contribution All authors were involved in conceptualization of study design and active management of patients. Dr. SJ and Dr. ASS were involved in collection and analysis of data. Dr. SJ, Dr. SG and Dr. GSB prepared the final manuscript which was read and approved by all the authors.

Funding No internal or external funding was availed for this study. 


\section{Compliance with Ethical Standards}

Conflict of interest The authors have no conflict of interest to declare.

\section{References}

1. Gupta A, Arora V, Nair D, Agrawal N, Su YX, Holsinger FC et al (2020) Status and strategies for the management of head and neck cancer during COVID-19 pandemic: Indian scenario. Head Neck 42(7):1460-1465

2. Mehanna H, Hardman JC, Shenson JA, Abou-Foul AK, Topf MC et al (2020) Recommendations for head and neck oncology practice in a setting of acute severe resource constraint during the COVID-19 pandemic: an international consensus. Lancet Oncol 21(7):e350-e359

3. Day DT, Sher DJ, Lee RC, Truelson JM, Myers LL, Sumer BD et al (2020) Head and neck oncology during COVID-19 pandemic: reconsidering traditional treatment paradigms in light of new surgical and other multilevel risks. Oral Oncol 105:104684

4. Nekkanti SS, Vasudevan Nair S, Parmar V, Saklani A, Shrikhande S, Sudhakar Shetty N et al (2020) Mandatory preoperative COVID-19 testing for cancer patients-is it justified? J Surg Oncol 122(7):1288-1292

5. Dindo D, Demartines N, Clavien PA (2004) Classification of surgical complications: a new proposal with evaluation in a cohort of 6336 patients and results of survey. Ann Surg 240:205-213

6. Brar S, Ofo E, Hyde N, Kim D, Odutoye T, Allin D et al (2020) Outcomes of elective head and neck confirmed or suspected cancer surgery during COVID-19 pandemic. Eur Arch Otorhinolaryngol. https://doi.org/10.1007/s00405-020-06194-2

7. Yanez Benitez C, Guemes A, Aranda J, Riberiro M, Ottolino P, Di Saverio $S$ et al (2020) Impact of personal protective equipment on surgical performance during the COVID-19 pandemic. World J Surg 44(9):2842-2847

Publisher's Note Springer Nature remains neutral with regard to jurisdictional claims in published maps and institutional affiliations. 